\title{
Electronic excitations of the chromophore from the fluorescent protein asFP595 in solutions
}

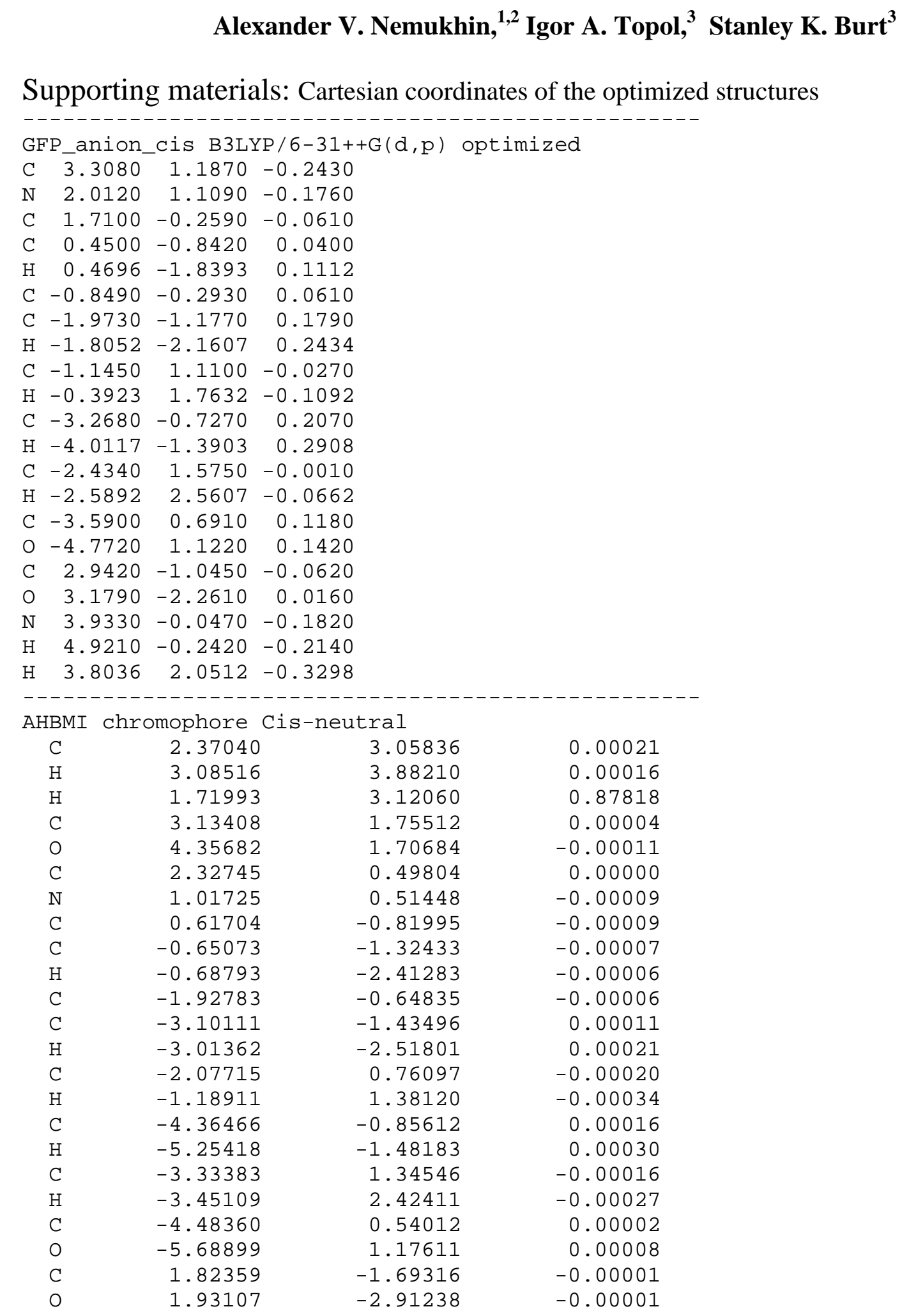




$\begin{array}{lrrr}\mathrm{N} & 2.88516 & -0.77366 & 0.00006 \\ \mathrm{C} & 4.28423 & -1.18719 & 0.00009 \\ \mathrm{H} & 4.27985 & -2.27878 & 0.00013 \\ \mathrm{H} & 4.80053 & -0.81451 & 0.88645 \\ \mathrm{H} & 1.71974 & 3.12068 & -0.87762 \\ \mathrm{H} & 4.80057 & -0.81456 & -0.88628 \\ \mathrm{H} & -6.41018 & 0.53219 & 0.00013 \\ -\end{array}$

\begin{tabular}{|c|c|c|c|}
\hline \multicolumn{4}{|c|}{ BMI $\quad \mathrm{cl}$} \\
\hline C & 3.54285 & 3.46687 & ๑.58971 \\
\hline $\mathrm{H}$ & 2.98743 & 3.58527 & 1.52527 \\
\hline $\mathrm{H}$ & 4.51108 & 3.96407 & 0.65810 \\
\hline C & 3.77014 & 2.00322 & $\odot .29248$ \\
\hline 0 & 4.88853 & 1.52097 & 0.16578 \\
\hline C & 2.55647 & 1.14881 & 0.15631 \\
\hline $\mathrm{N}$ & 1.34842 & 1.62636 & 0.28575 \\
\hline C & 0.47056 & ๑. 55322 & ๑. .09703 \\
\hline C & -0.87962 & $\odot .77767$ & 0.18004 \\
\hline $\mathrm{H}$ & -1.10149 & 1.82239 & 0.39434 \\
\hline C & -2.04846 & -0.05867 & 0.04642 \\
\hline C & $-3.307 \odot 7$ & 0.57075 & 0.20635 \\
\hline $\mathrm{H}$ & -3.34409 & 1.63576 & 0.41888 \\
\hline C & -2.03886 & -1.45003 & -0.23007 \\
\hline $\mathrm{H}$ & -1.08886 & -1.95621 & -0.35702 \\
\hline C & -4.49577 & -0.13713 & ๑. 09865 \\
\hline $\mathrm{H}$ & -5.44967 & ๑. 36951 & 0.22559 \\
\hline C & -3.22390 & -2.16174 & -0.33846 \\
\hline $\mathrm{H}$ & -3.21653 & -3.22590 & $-\odot .54978$ \\
\hline C & -4.45613 & -1.51226 & -0.17541 \\
\hline 0 & -5.58206 & -2.26896 & -0.29441 \\
\hline C & 1.27468 & -0.67382 & -0.17006 \\
\hline 0 & ๑.94908 & -1.83834 & -0.39266 \\
\hline $\mathrm{N}$ & 2.59365 & -0.21633 & -0.11650 \\
\hline C & 3.73808 & -1.09719 & -0.32499 \\
\hline $\mathrm{H}$ & 4.37954 & -1.11546 & 0.55780 \\
\hline $\mathrm{H}$ & 3.33015 & -2.09171 & -0.51309 \\
\hline $\mathrm{H}$ & 4.33125 & -0.76683 & -1.17940 \\
\hline $\mathrm{H}$ & 2.93296 & 3.92656 & -0.19411 \\
\hline $\mathrm{H}$ & -6.37059 & -1.72477 & -0.16430 \\
\hline
\end{tabular}

\begin{tabular}{|c|c|c|c|}
\hline \multicolumn{4}{|c|}{ AHBMI c } \\
\hline C & 2.44628 & 3.04045 & $\odot .00 \odot 22$ \\
\hline $\mathrm{H}$ & 2.76549 & 3.60805 & -0.88101 \\
\hline $\mathrm{H}$ & 2.76339 & 3.60653 & $\odot .88320$ \\
\hline C & 3.16456 & 1.70190 & $-\odot .0 \odot \odot \odot 9$ \\
\hline 0 & 4.40371 & 1.65668 & $-\odot . \odot \odot \odot 17$ \\
\hline C & 2.34355 & 0.49787 & $-\odot .0 \odot \odot \odot 6$ \\
\hline $\mathrm{N}$ & 1.01668 & $\odot .51626$ & $-\odot .0 \odot \odot \odot 7$ \\
\hline C & 0.59853 & -0.78949 & $-\odot .0 \odot \odot \odot \odot$ \\
\hline C & -0.71931 & -1.27179 & $0.000 \odot 2$ \\
\hline $\mathrm{H}$ & $-\odot .75946$ & -2.36127 & ๑.00005 \\
\hline C & -1.95562 & $-\odot .61436$ & ๑. $00 \odot 01$ \\
\hline C & -3.16174 & -1.40122 & $\odot .00003$ \\
\hline $\mathrm{H}$ & -3.05886 & -2.48586 & $\odot .00005$ \\
\hline C & -2.12042 & ๑. 81692 & $-\odot .0 \odot \odot \odot 2$ \\
\hline $\mathrm{H}$ & -1.22468 & 1.42862 & $-\odot .0 \odot \odot \odot 3$ \\
\hline C & -4.40676 & $-\odot .83818$ & $\odot .0 \odot \odot \odot 2$ \\
\hline
\end{tabular}




\begin{tabular}{|c|c|c|c|}
\hline $\mathrm{H}$ & -5.30527 & -1.44981 & $\odot .0 \odot \odot \odot 3$ \\
\hline C & -3.35864 & 1.39519 & $-\odot . \odot \odot \odot \odot 3$ \\
\hline $\mathrm{H}$ & -3.47183 & 2.47649 & $-\odot .0 \odot \odot \odot 5$ \\
\hline C & -4.59298 & 0.61042 & $-\odot .0 \odot \odot \odot 1$ \\
\hline 0 & -5.72772 & 1.14340 & $-\odot .0 \odot \odot \odot 2$ \\
\hline C & 1.76088 & -1.68819 & ๑. 00003 \\
\hline 0 & 1.84799 & -2.92499 & $\odot . \odot \odot \odot \odot 9$ \\
\hline $\mathrm{N}$ & 2.84890 & $-\odot .80390$ & $-\odot .0 \odot \odot \odot 2$ \\
\hline C & 4.22689 & -1.25955 & $\odot .00 \odot \odot 2$ \\
\hline $\mathrm{H}$ & 4.18763 & -2.35177 & 0.00015 \\
\hline $\mathrm{H}$ & 4.76071 & $-\odot .9 \odot 209$ & 0.88426 \\
\hline $\mathrm{H}$ & 1.36218 & 2.93028 & - ๑. .०102 \\
\hline $\mathrm{H}$ & 4.76069 & -0.90230 & -0.88431 \\
\hline \multicolumn{4}{|c|}{ AHBMI chromophore Trans-anion } \\
\hline C & 2.75543 & 3.91066 & 1.28558 \\
\hline $\mathrm{H}$ & 3.11107 & 4.14979 & 2.29414 \\
\hline $\mathrm{H}$ & 3.19325 & 4.64862 & 0.60390 \\
\hline C & 3.27245 & 2.53283 & $\odot .90396$ \\
\hline 0 & 4.49160 & 2.30396 & $\odot .89495$ \\
\hline C & 2.28579 & 1.52133 & 0.55827 \\
\hline $\mathrm{N}$ & $\odot .97880$ & 1.73880 & 0.55966 \\
\hline C & $\odot .36170$ & $\odot .56740$ & $\odot .18467$ \\
\hline C & -1.04736 & 0.55135 & 0.11243 \\
\hline $\mathrm{H}$ & -1.45335 & 1.52723 & 0.38082 \\
\hline C & -2.01174 & $-\odot .40632$ & $-\odot .21622$ \\
\hline C & -3.39733 & $-\odot .00337$ & $-\odot .16370$ \\
\hline $\mathrm{H}$ & -3.61542 & 1.02349 & 0.12877 \\
\hline C & -1.75323 & -1.77170 & -0.60663 \\
\hline $\mathrm{H}$ & $-\odot .71901$ & -2.09869 & $-\odot .65354$ \\
\hline C & -4.42285 & -0.85217 & -0.46320 \\
\hline $\mathrm{H}$ & -5.45942 & $-\odot .52808$ & -0.41723 \\
\hline C & -2.77254 & -2.63037 & $-\odot .90875$ \\
\hline $\mathrm{H}$ & -2.56553 & -3.65672 & -1.20161 \\
\hline C & -4.17923 & -2.23792 & -0.86034 \\
\hline 0 & -5.11195 & -3.02754 & -1.13797 \\
\hline C & 1.38237 & -0.46054 & $-\odot .06957$ \\
\hline 0 & 1.31566 & -1.64896 & -0.42337 \\
\hline $\mathrm{N}$ & 2.58502 & ๑. 20985 & 0.18576 \\
\hline C & 3.87789 & -0.43784 & ๑. 05646 \\
\hline $\mathrm{H}$ & 4.41719 & -0.43290 & 1.00717 \\
\hline $\mathrm{H}$ & 3.67584 & -1.46487 & $-\odot .25677$ \\
\hline $\mathrm{H}$ & 4.49863 & 0.06810 & $-\odot .68740$ \\
\hline $\mathrm{H}$ & 1.66750 & 3.96895 & 1.24993 \\
\hline \multicolumn{4}{|c|}{ AHBMI chromophore Cis-zwitterion } \\
\hline C & 2.77876 & 2.65517 & 0.82865 \\
\hline $\mathrm{H}$ & 2.24322 & 2.49206 & 1.77179 \\
\hline $\mathrm{H}$ & 3.60166 & 3.34670 & 1.01194 \\
\hline C & 3.36409 & 1.36149 & 0.28899 \\
\hline 0 & 4.57406 & 1.20724 & 0.14089 \\
\hline C & 2.43567 & 0.28192 & $-\odot .05609$ \\
\hline $\mathrm{N}$ & 1.09169 & 0.34961 & 0.07599 \\
\hline C & 0.47952 & -0.82703 & -0.30060 \\
\hline C & -0.88312 & -1.18031 & $-\odot .2709 \odot$ \\
\hline $\mathrm{H}$ & -1.01816 & -2.24049 & -0.47672 \\
\hline C & -2.03050 & -0.44100 & -0.01920 \\
\hline
\end{tabular}




\begin{tabular}{|c|c|c|c|}
\hline C & -3.29020 & -1.14902 & ๑. 10109 \\
\hline H & -3.27502 & -2.22719 & -0.04137 \\
\hline C & -2.08158 & $\odot .99879$ & 0.13126 \\
\hline $\mathrm{H}$ & -1.19117 & 1.59298 & $-\odot .05679$ \\
\hline C & -4.45378 & $-\odot .51989$ & 0.40753 \\
\hline $\mathrm{H}$ & -5.38643 & -1.06519 & ๑. 51257 \\
\hline C & -3.23561 & 1.65035 & 0.42741 \\
\hline H & -3.27314 & 2.73191 & ๑. 51571 \\
\hline C & -4.50752 & $\odot .93137$ & ๑. 61505 \\
\hline 0 & -5.55290 & 1.52715 & 0.91053 \\
\hline C & 1.54893 & -1.72200 & -0.71409 \\
\hline 0 & 1.52001 & -2.87511 & -1.13017 \\
\hline $\mathrm{N}$ & 2.73846 & -0.94980 & $-\odot .54295$ \\
\hline C & 4.05689 & -1.48817 & $-\odot .87136$ \\
\hline $\mathrm{H}$ & 4.69217 & -1.51174 & ๑. 01508 \\
\hline $\mathrm{H}$ & 3.88368 & -2.49821 & -1.24458 \\
\hline $\mathrm{H}$ & $\odot .61735$ & 1.13997 & 0.48627 \\
\hline $\mathrm{H}$ & 2.08612 & 3.11508 & 0.11385 \\
\hline $\mathrm{H}$ & 4.53964 & $-\odot .87558$ & -1.63401 \\
\hline \multicolumn{4}{|c|}{ AHBMI chromophore Trans-zwitterion } \\
\hline C & 3.41075 & 3.61658 & $-\odot .14279$ \\
\hline $\mathrm{H}$ & 2.88142 & 3.91603 & 0.76986 \\
\hline $\mathrm{H}$ & 4.36324 & 4.14592 & -0.18218 \\
\hline C & 3.70224 & 2.12465 & $-\odot .15667$ \\
\hline 0 & 4.85156 & 1.68999 & $-\odot .20628$ \\
\hline C & 2.56463 & 1.20913 & -0.10892 \\
\hline $\mathrm{N}$ & 1.26858 & 1.58074 & -0.05283 \\
\hline C & $\odot .40635$ & $\odot .50114$ & -0.01694 \\
\hline C & -0.99339 & $\odot .72359$ & 0.04327 \\
\hline $\mathrm{H}$ & -1.24729 & 1.78506 & 0.05506 \\
\hline C & -2.08811 & -0.12258 & ๑. . 08948 \\
\hline C & -3.40339 & $\odot .49621$ & 0.14634 \\
\hline $\mathrm{H}$ & -3.45382 & 1.58363 & 0.14940 \\
\hline C & -2.03607 & -1.57415 & 0.08613 \\
\hline $\mathrm{H}$ & -1.06375 & -2.05243 & 0.04405 \\
\hline C & -4.54369 & -0.23368 & 0.19461 \\
\hline $\mathrm{H}$ & -5.52275 & ๑.23357 & 0.23694 \\
\hline C & -3.17234 & -2.31509 & 0.13424 \\
\hline $\mathrm{H}$ & -3.13602 & -3.40036 & 0.13186 \\
\hline C & -4.50875 & -1.70502 & ๑.19199 \\
\hline 0 & -5.54422 & -2.38329 & 0.23589 \\
\hline C & 1.26141 & -0.67759 & -0.05497 \\
\hline 0 & 1.01390 & -1.88274 & $-\odot .04563$ \\
\hline $\mathrm{N}$ & 2.58355 & -0.15124 & -0.11117 \\
\hline C & 3.75201 & -1.02786 & $-\odot .16255$ \\
\hline $\mathrm{H}$ & 4.39914 & -0.85075 & 0.69753 \\
\hline $\mathrm{H}$ & 3.36444 & -2.04688 & -0.14676 \\
\hline $\mathrm{H}$ & 4.32243 & -0.84910 & -1.07502 \\
\hline $\mathrm{H}$ & 2.80688 & 3.91786 & -1.00727 \\
\hline H & $\odot .96485$ & 2.54415 & $-\odot .03880$ \\
\hline
\end{tabular}

AHBMI chromophore and 7 water molecules: Structure A
$\begin{array}{llll}\text { C } \quad 3.4970 & 3.6740 & 0.8620\end{array}$
H $\quad 3.2040 \quad 4.0970-0.1060$
$\begin{array}{llll}\text { H } & 2.7340 & 3.9750 & 1.5850\end{array}$
$\begin{array}{lll}\text { C } \quad 3.5950 & 2.1720 & 0.7630\end{array}$ 


\begin{tabular}{|c|c|c|c|}
\hline & 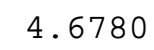 & & \\
\hline & $2 \quad 3730$ & & \\
\hline & & & \\
\hline & & & \\
\hline & 1. & & \\
\hline & 1. & & \\
\hline & & & \\
\hline & & & \\
\hline & & & \\
\hline & & & \\
\hline & -1. & & \\
\hline & -4 & & \\
\hline & & & \\
\hline & -3 & & \\
\hline & -3 & & \\
\hline & -4 & -0 & \\
\hline & -5 & & \\
\hline & & & \\
\hline & & & \\
\hline & & & \\
\hline & & & \\
\hline & & -1 & \\
\hline & & & \\
\hline & & & \\
\hline & & & \\
\hline & -8. & & \\
\hline & -7. & & \\
\hline & -8 & $-\odot$ & \\
\hline & -6. & -3 & \\
\hline & & & \\
\hline & & & \\
\hline & & & \\
\hline & & -4 & \\
\hline & & & \\
\hline & & & \\
\hline & & & \\
\hline & & & \\
\hline & & & \\
\hline & & & \\
\hline & & & \\
\hline & & & \\
\hline $\mathrm{H}$ & & & \\
\hline & & & \\
\hline & & & \\
\hline & & & \\
\hline & & & \\
\hline
\end{tabular}

AHBM chromophore and 7 water molecules: Structure

AHBMI chromophore and 7 water molecules: Structure B

$\begin{array}{rrrr}\mathrm{C} & 4.2350 & 2.8670 & 0.1290 \\ \mathrm{H} & 3.5030 & 3.4560 & -0.4260 \\ \mathrm{H} & 4.1330 & 3.1310 & 1.1890 \\ \mathrm{C} & 4.0190 & 1.3840 & -0.0370 \\ \mathrm{O} & 4.9870 & 0.6090 & -0.1560 \\ \mathrm{C} & 2.6490 & 0.8780 & -0.0340 \\ \mathrm{~N} & 1.5940 & 1.6480 & 0.1710 \\ \mathrm{C} & 0.4730 & 0.8410 & 0.1210 \\ \mathrm{C} & -0.7880 & 1.4310 & 0.3150\end{array}$




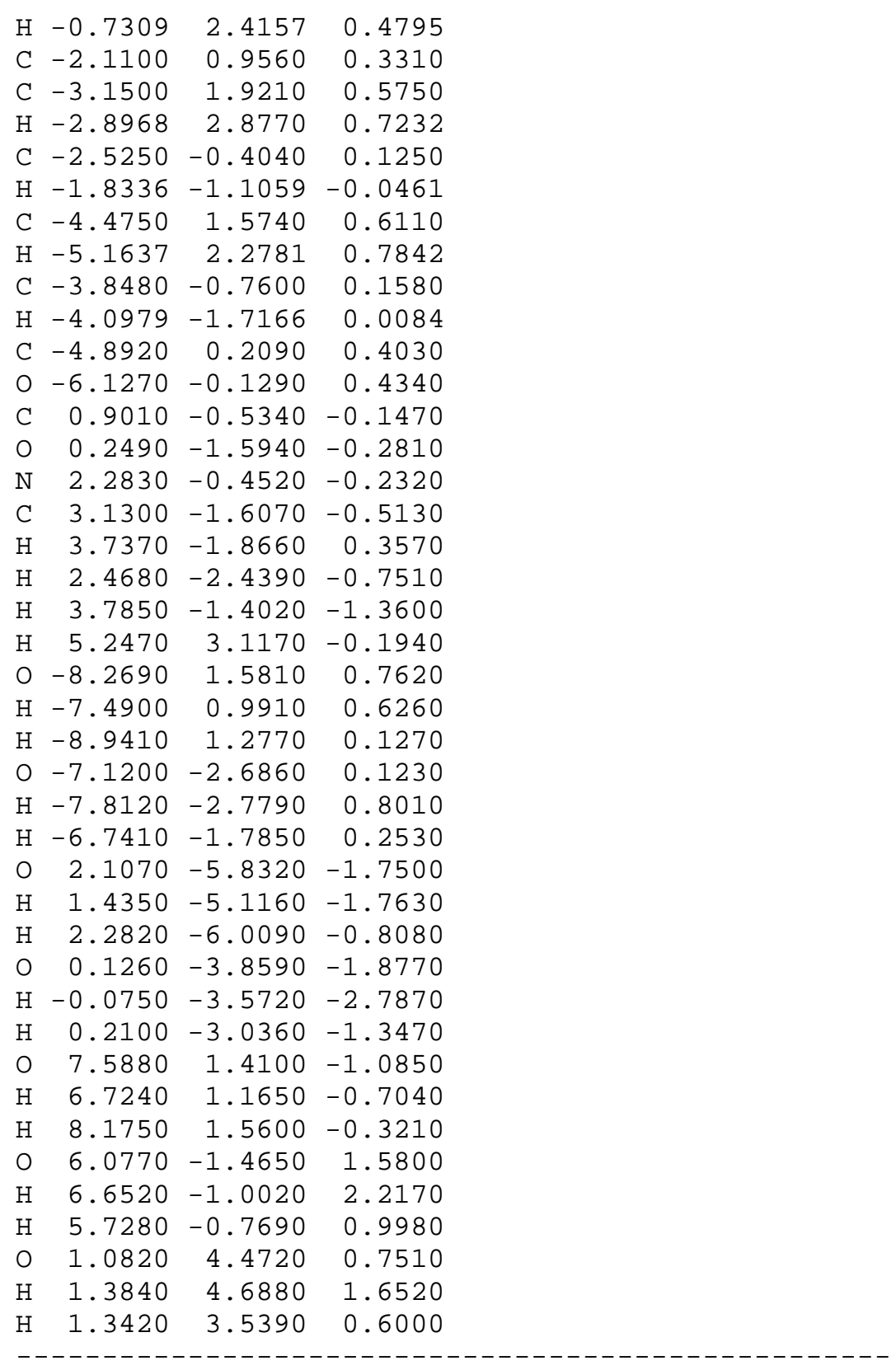

